\title{
Obsessive-compulsive symptoms in Parkinson's disease
}

\author{
M Alegret, C Junqué, F Valldeoriola, P Vendrell, M J Martí, E Tolosa
} d'Hebron 171, 08035 Barcelona, Spain M Alegret

C Junqué

$P$ Vendrell

Parkinson's and Movement Disorders Unit, Institut Clínic de Malalties del Sistema Nerviós (ICMSN),

Department of Neurology, Hospital Clinic Universitari, School of Medicine F Valldeoriola M J Martí

E Tolosa

Correspondence to: Professor C Junqué cjunque@psi.ub.es

Received 6 July 2000 and in revised form

19 October 2000

Accepted 10 November 2000

Keywords: Parkinson's disease; obsessive-compulsive disorder; basal ganglia

Several structural and functional neuroimaging studies have shown that obsessive-compulsive disorder is related to dysfunction of the basal ganglia. ${ }^{12}$ Lesions in the basal ganglia produce obsessive-compulsive symtomatology similar to idiopathic obsessive-compulsive disorder. ${ }^{3}$ In addition, the incidence of obsessivecompulsive disorder is high in Huntington's disease and Tourette's syndrome. ${ }^{4-6}$

It is well known that patients with Parkinson's disease manifest several dysfunctions of frontobasal ganglia circuitry. Dysfunction in the limbic circuitry may also be responsible for the occurrence of obsessive-compulsive traits in patients with Parkinson's disease. Tomer et $a l^{7}$ found a relation between obsessivecompulsive symptomatology and the severity of motor impairment in Parkinson's disease.

\begin{abstract}
Department of Psychiatry and Clinical

Psychobiology, University of Barcelona, Institut d'Investigacions Biomèdiques August Pi i Sunyer, Pg Vall

Abstract

To systematically investigate obsessivecompulsive traits in Parkinson's disease, patients were administered the Maudsley obsessional-compulsive inventory (MOCI) and a modification of the Leyton obsessional inventory (LOI) to a sample of nondemented and non-depressed patients with Parkinson's disease. Patients with severe Parkinson's disease showed more obsessive traits than normal controls in MOCI and LOI total scores, and in the "checking", "doubting", and "cleaning" subscales of the MOCI. By contrast, patients with mild disease did not differ from controls. A significant correlation was found between severity and duration of illness and MOCI total score. These results support the involvement of basal ganglia in obsessivecompulsive symptomatology. As patients with mild Parkinson's disease did not differ from controls, obsessive-compulsive disorder does not seem to be directly related to the initial nigrostriatal dopaminergic deficiency which causes clinical Parkinson's disease symptomatology. The appearance of obsessive symptoms could be related to the subset of neurochemical changes taking place at the level of the basal ganglia circuitry as disease progresses. (F Neurol Neurosurg Psychiatry 2001;70:394-396)
\end{abstract}

However, in a group of 16 patients with Parkinson's disease Müller et al did not find differences between patients and normal controls in the Maudsley obsessional-compulsive inventory (MOCI). In a previous study, we reported that patients with Parkinson's disease who had undergone pallidotomy had improved scores on the MOCI. ${ }^{9}$

The aim of the present study was to systematically investigate obsessive-compulsive traits in Parkinson's disease. Two questionnaires were used: the MOCI, mainly applied in idiopathic obsessive-compulsive disorder, ${ }^{10}$ and a modified version of Leyton obsessional inventory (LOI), which was reported to be sensitive to Tourette's syndrome. ${ }^{6}$

\section{Methods}

SUBJECTS

The MOCI was administered to 72 consecutive non-demented patients with idiopathic Parkinson's disease (36 men and 36 women) from the Department of Neurology at the Hospital Clinic Universitari in Barcelona. The control group comprised 72 subjects without history of neurological or psychiatric illness (36 men and 36 women). They were patients' spouses or friends, recruited from the neurology outpatients' department of the Hospital Clinic Universitari. The groups were matched by age, sex, and education. The mean (SD) age of the patients was 63.25 (8.58), educational level 8.56 (4.32) years; for controls, the mean (SD) age was 63.63 (10.13), educational level 9.39 (4.31) years. Mean (SD) age at onset of Parkinson's disease was 51.90 (10.65) years, duration of disease 11.51 (7.53) years, and mean Hoehn and Yahr stage 3.03 (SD 1.12).

A modified version of the LOI was administered to a subgroup of 54 patients with Parkinson's disease ( 27 men, 27 women) and 54 normal controls (27 men, 27 women) from the sample. Mean (SD) patient age was 64.13 years (8.91), educational level 8.85 (4.44) years, duration of illness 10.65 (8.00) years, and mean Hoehn and Yahr stage 2.91 (1.19). The control group was also matched to the Parkinson's disease group by mean (SD) age (64.15 (10.51)), education (9.39 (4.45) years), and sex (27 men, 27 women). 
Comparison between mild Parkinson's disease (PD), severe PD, and control groups in MOCI and LOI questionnaires

\begin{tabular}{llllll}
\hline & Mild PD & Severe PD & Controls & F Value & Post hoc \\
\hline Group 1: & $(\mathrm{n}=25)$ & $(\mathrm{n}=47)$ & $(\mathrm{n}=72)$ & $(2,141)$ & \\
MOCI total & $4.12(3.15)$ & $7.62(4.19)$ & $4.83(3.57)$ & $10.412^{\star \star \star}$ & $\dagger \neq$ \\
MOCI checking & $1.08(1.29)$ & $2.23(2.01)$ & $1.26(1.74)$ & $5.326^{\star \star}$ & $\ddagger$ \\
MOCI cleaning & $1.28(1.43)$ & $1.87(1.41)$ & $1.21(1.37)$ & $3.417^{\star}$ & $\dagger \neq$ \\
MOCI doubting & $1.48(1.36)$ & $3.09(1.60)$ & $2.18(1.48)$ & $10.331^{\star \star \star}$ & $\dagger \neq$ \\
MOCI slowness & $2.28(0.61)$ & $2.32(1.07)$ & $2.14(0.74)$ & 0.724 & \\
Group 2: & $(\mathrm{n}=23)$ & $(\mathrm{n}=31)$ & $(\mathrm{n}=54)$ & $(2,105)$ & \\
LOI & $48.04(29.04)$ & $62.03(28.18)$ & $48.41(22.75)$ & $3.14^{\star}$ & $\dagger$ \\
LOI TS items & $1.30(2.18)$ & $2.03(3.05)$ & $1.44(1.79)$ & 0.86 & \\
LOI OCD items & $16.43(11.55)$ & $21.06(9.27)$ & $18.31(8.37)$ & 1.70 & \\
\end{tabular}

Values are means $(\mathrm{SD})$

${ }^{\star} \mathrm{p}<0.05 ;{ }^{\star \star} \mathrm{p}<0.01 ;{ }^{\star \star \star} \mathrm{p}<0.001$ from controls

†Significant differences between mild PD and severe PD.

$¥$ Significant differences between severe PD and normal controls.

TS=Tourette's syndrome; $\mathrm{OCD}=$ obsessive-compulsive disorder.

ASSESSMENT OF OBSESSIVE-COMPULSIVE TRAITS

The MOCI comprises 30 sentences which are classified into four factors: checking (nine items), cleaning (11 items), doubting (seven items), and slowness (seven items). Subjects were instructed to answer true or false. The inventory includes both affirmative and negative sentences.

The modified version of the LOI, proposed by Frankel et $a l^{6}{ }^{6}$ was constructed from questions derived from the original LOI, ${ }^{11}$ supplemented by additional questions designed to elicit obsessive-compulsive disorder phenomena. The LOI consists of 40 statements, and subjects were asked to make a graded response indicating whether the statement was true for his or her symptoms: "never" (score 0), "rarely" (1), "sometimes" (2), "moderately often" (3), "frequently" (4) or "always" (5). Frankel et $a l^{6}$ identified a group of items (14, $19,21,24,29,38$, and 40) as preferentially endorsed by patients with Tourette's symdrome and 11 questions ( 9 to $12,15,23,25$, $28,30,34$, and 37 ) that elicited high scores from patients with idiopathic obsessivecompulsive disorder.

\section{Results}

Patients with Parkinson's disease obtained a higher total MOCI score than normal controls $(t=2.42, \mathrm{p}=0.017)$. As MOCI total score correlated with Hoehn and Yahr stage $(r=0.33$, $\mathrm{p}=0.004)$ and duration of illness $(r=0.34$, $\mathrm{p}=0.005$ ), two subgroups were created according to severity of Parkinson's disease: mild (Hoehn and Yahr \$2.5) and severe (Hoehn and Yahr>2.5). One way analysis of variance (ANOVA) showed a significant effect of group $(F=10.41, \mathrm{p}=0.0001)$. Post hoc analysis using Duncan's procedure showed higher scores in patients with severe Parkinson's disease than in those with mild Parkinson's disease and normal controls (table). The analysis of MOCI factors showed a significant group effect on the "checking", "doubting" and "cleaning" subscales (analysis of variance (ANOVA), $F=5.33$, $\mathrm{p}=0.0059 ; F=10.33, \mathrm{p}=0.0001 ;$ and $F=3.42$, $\mathrm{p}=0.0355$, respectively).

The LOI scores also disclosed significant differences between groups. The obsessional scores for patients with severe Parkinson's disease were higher than those for patients with mild Parkinson's disease and controls. Taking a cut off level of 70 to determine a definitely raised inventory score ${ }^{6} 13$ of the 54 patients $(24.1 \%)$ and 10 of the 54 control subjects $(18.5 \%)$ scored in this range. Total scores for MOCI and LOI were strongly correlated in both patients with Parkinson's disease $(r=0.70$, $\mathrm{p}=0.000)$ and controls $(\mathrm{r}=0.65, \mathrm{p}=0.000)$. Leyton total score was not related to age $(r=0.15, \mathrm{p}=0.288)$, Hoehn and Yahr $(r=0.02$, $\mathrm{p}=0.885)$ or duration of illness $(r=0.05$, $\mathrm{p}=0.729)$.

\section{Discussion}

Relations between Parkinson's disease and obsessive-compulsive disorder have been previously suggested in other studies, ${ }^{12}{ }^{13}$ but few researchers have systematically investigated this contention. Hardie et $a l^{13}$ described complex mannerisms and organised rituals in conjunction with the on-off phenomenon in patients with Parkinson's disease, but only two studies have hitherto administered obsessivecompulsive questionnaires. Tomer et al compared data from 30 patients with Parkinson's disease with the normative data from Leyton's original questionnaire. They found that out of 30 patients, 17 had a higher symptom score, and 19 a higher trait score than the mean of normal controls. The authors concluded that obsessive-compulsive symptoms may be an important but unrecognised feature in some patients with idiopathic Parkinson's disease. Müller $e t a l^{8}$ reported negative results using the MOCI and the Hamburg obsessive-compulsive inventory in a sample of 20 patients with Parkinson's disease and 43 controls. The small sample size and the heterogeneity of Parkinson's disease may be responsible for the lack of statistical significance in this study.

Patients with severe Parkinson's disease presented significantly more self reported obsessive-compulsive symptoms than controls in both questionnaires administered, but none of the patients was diagnosed as having obsessive-compulsive disorder according to DSM-IV criteria, and none were receiving psychopharmacological treatment for obsessivecompulsive disorder symptomatology. Although the MOCI and LOI scales are widely accepted as descriptive self estimation scales for obsessive-compulsive symptoms, no diagnostic value is given.

Our patients with severe Parkinson's disease differed from controls in almost all scales (checking, cleaning, and doubting). Patients with mild Parkinson's disease had no obsessivecompulsive symptoms. In addition, a correlation between years of evolution and MOCI global score was found. Thus, the present study showed that obsessive-compulsive symptoms appeared late during the disease progression in patients with idiopathic Parkinson's disease. This fact suggests that the emergence of obsessive symptoms could be directly related to the subset of neurochemical changes taking place at the level of the basal ganglia circuitry as the disease progresses. $^{14}$ The functional disturbances produced by degeneration of the nigrostriatal pathway could influence the striatofrontal circuits in the advanced stages of 
Parkinson's disease. Another alternative explanation could be that some of the patients in our study presented direct frontocortical damagethat is, gliosis, neuronal loss, and Lewy bodies in the cytoplasm. However, this explanation is unlikely as the patients involved in this study showed no signs of dementia, hallucinations, or any other symptomatology of Lewy body dementia. ${ }^{15}$

Obsessive-compulsive disorder is mainly seen in degenerative processes such as Huntington's disease ${ }^{4}$ and is associated with Tourette's syndrome. ${ }^{5}$ The motor symptoms of Parkinson's disease are in some ways the opposite to Tourette's syndrome and Huntington's disease, as in these two diseases there is a motor overactivation. However, over time levodopa treatment in Parkinson's disease is able to induce dyskinesias. In patients with very advanced Parkinson's disease who underwent pallidotomy we found an improvement of both obsessive-compulsive traits and dyskinesias in the assessment performed 3 months after surgery, ${ }^{9}$ suggesting that their mechanisms are similar. Litvan et $a l^{16}$ found that hyperkinetic syndromes, such as Huntington's disease, are associated with hyperactive behaviours. They suggested that these behaviours are secondary to an excitatory subcortical output through the medial and orbitofrontal cortical circuits.

Several models of obsessive-compulsive disorder-and other psychiatric diseases such as melancholia-centre around the possibility that symptoms represent behavioural programmes that are pathologically and repetitively generated by dysfunctional basal ganglia circuits, as in motor dysfunction. ${ }^{17}{ }^{18}$ Lesional data in Parkinson's disease also point to a link between obsessive-compulsive disorder and basal ganglia. Daniele et $a l^{19}$ reported a case of idiopathic Parkinson's disease which started obsessive thoughts and compulsions after a vascular unilateral lesion restricted to the left putamen. Obsessions appeared after an interval of days, compulsions after a few weeks, and both symptoms progressed over subsequent months. This patient was classified as very severe on the Yale-Brown obsessive-compulsive scale.

Analysing the items that Frankel et $a l^{6}$ identified as preferentially endorsed by patients with Tourette's syndrome, we did not find differences between patients with Parkinson's disease and controls. The classic obsessivecompulsive disorder symptoms found in Tourette's syndrome (questions which involved blurting obscenities, imitating the movements of others, counting compulsions, and impulses to hurt oneself) were not found in our Parkinson's disease sample. Slowness might be expected to be characteristic of Parkinson's disease from a clinical point of view, but the
MOCI score on this subscale (slowness and repetition) did not increase.

It has been suggested that patients with Parkinson's disease display a specific cluster of personality traits consisting of increased rigidity, conscientiousness, industriousness, orderliness, and cautiousness. Menza et $a l^{20}$ reported that patients with Parkinson's disease were less "novelty seeking" than controls. Low novelty seeking patients were described as being reflective, rigid, stoic, slow tempered, frugal, orderly, and persistent. Some of these are obsessive traits.

In summary, our data support the involvement of basal ganglia in obsessive-compulsive symptomatology.

This study is partially supported by grants 1997FI00147 and 99SGR00081 from the Generalitat of Catalunya. We thank Chris Summerfield for his English revision.

1 Robinson D, Wu H, Munne RA, et al. Reduced caudate nucleus volume in obsessive-compulsive disorder. Arch Gen Psychiatry 1995;52:393-8.

2 Baxter LR, Phelps ME, Mazziotta JC, et al. Local cerebral glucose metabolic rates in obsessive-compulsive disorder: a comparison with rates in unipolar depression and in normal controls. Arch Gen Psychiatry 1987;44:211-18.

3 Laplane D, Lavasseur M, Pillon B, et al. Obsessivecompulsive and other behavioural changes with bilateral basal ganglia lesions. A neuropsychological magnetic resonance imaging and positron tomography study. Brain 1989;112:699-725.

4 Cummings JL. Frontal-subcortical circuits and human behavior. Arch Neurol 1993;50:873-80.

5 Cummings JL, Frankel M. Gilles de la Tourette syndrome and the neurological basis of obsessions and compulsions. Biol Psychiatry 1985;20:1117-26.

6 Frankel M, Cummings JL, Robertson MM, et al. Obsessions and compulsions in Gilles de la Tourette's syndrome. Neurology 1986;36:378-82.

7 Tomer R, Levin BE, Weiner WJ. Obsessive-compulsive symptoms and motor asymmetries in Parkinson's disease. Neuropsychiatry Neuropsychol Behav Neurol 1993;6:26-30.

8 Müller N, Putz A, Kathmann N, et al. Characteristics of obsessive-compulsive symptoms in Tourette's syndrome, obsessive-compulsive disorder, and Parkinson's disease. Psychiatr Res 1997;70:105-14.

9 Junqué C, Alegret $\mathrm{M}$, Nobbe FA, et al. Cognitive and behavioral changes after unilateral posteroventral pallidotomy: relationship with lesional data from MRI. Mov Disord 1999;5:780-9.

10 Hodgson RJ, Rachman S. Obsessional-compulsive complaints. Behav Res Ther 1977;15:389-95.

11 Cooper J. The Leyton obsessional inventory. Psychol Med 1970;1:48-64.

12 Lees AJ. The neurobehavioural abnormalities in Parkinson's disease and their relationship to psychomotor retardation and obsessional compulsive disorders. Behav Neurol 1989; $2: 1-11$

13 Hardie RJ, Lees AJ, Stern GM. On-off fluctuations in Parkinson's disease. Brain 1984;107:487-506.

14 Lang AE, Lozano AM. Parkinson's disease. N Engl f Med 1998;339:1130-43.

15 Lopez OL, Litvan I, Catt KE, et al. Accuracy of four clinical diagnostic criteria for the diagnosis of neurodegenerative dementias. Neurology 1999;53:1292-9.

16 Litvan I, Paulsen JS, Mega MS, et al. Neuropsychiatric assessment of patients with hyperkinetic and hypokinetic movement disorders. Arch Neurol 1998;55:1313-19.

17 Saint-Cyr JA, Taylor AE, Nicholson K. Behavior and the basal ganglia. Adv Neurol 1995;65:1-28.

18 Austin MP, Mitchell P. The anatomy of melancholia: does frontal-subcortical pathophysiology underpin its psychofrontal-subcortical pathophysiology underpin its psycho-
motor and cognitive manifestations? Psychol Med 1995;25: motor an

19 Daniele A, Bartolomeo P, Cassetta E, et al. Obsessivecompulsive behaviour and cognitive impairment in a parkinsonian patient after left putaminal lesion. I Neurol Neurosurg Psychiatry 1997;62:288-9.

20 Menza MA, Golbe LI, Cody RA, et al. Dopamine-related personality traits in Parkinson's disease. Neurology 1993;43: 505-8. 